\title{
Improvement and Simulation of an Autonomous Time Synchronization Algorithm for a Layered Satellite Constellation
}

\author{
Feijiang Huang, ${ }^{1,2}$ Xiaochun Lu, ${ }^{2,3}$ Guangcan Liu, ${ }^{1}$ Liping Sun, \\ Wang Sheng, ${ }^{1}$ and Yingde Wang ${ }^{1}$ \\ ${ }^{1}$ Department of Electronics and Communication Engineering, Changsha University, Changsha 410022, China \\ ${ }^{2}$ Key Laboratory of Precision Navigation and Timing Technology, Chinese Academy of Sciences, Xian 710600, China \\ ${ }^{3}$ National Time Service Center, Chinese Academy of Sciences, Xian 710600, China \\ Correspondence should be addressed to Feijiang Huang; ccsuhf@@163.com
}

Received 1 September 2013; Revised 5 November 2013; Accepted 14 November 2013

Academic Editor: Cao Su-Qun

Copyright (C) 2013 Feijiang Huang et al. This is an open access article distributed under the Creative Commons Attribution License, which permits unrestricted use, distribution, and reproduction in any medium, provided the original work is properly cited.

\begin{abstract}
Autonomous time synchronization for satellite constellations is a key technology to establish a constellation system time without the use of a ground station. The characteristics of satellite visibility time for layered satellite constellations containing geostationary earth orbit (GEO), inclined geosynchronous orbit (IGSO), and medium earth orbit (MEO) satellites are simulated by establishing a visible satellite model. Based on the satellite visible simulation results for a layered constellation, this study investigates the autonomous time synchronization algorithm that corresponds to the layered constellation structure, analyzes the main error of the time synchronization algorithm, and proposes methods to improve the characteristics of satellite movement in the constellation. This study uses an improved two-way time synchronization algorithm for autonomous time synchronization in the GEO-MEO satellite layer of a layered satellite constellation. The simulation results show that in a condition with simulation errors, the time synchronization precision of this improved algorithm can be controlled within $5 \mathrm{~ns}$ and used in high-precision autonomous time synchronization between layered satellite constellations.
\end{abstract}

\section{Introduction}

With the development of space science and technology, aerospace application systems require improvements in terms of high-precision time frequency, which is used in satellite navigation, space-based integrated information networks, space-based measurement and control, distributed-type satellite systems, and deep space detection. In current aerospace applications, time reference is established on the ground. Thus, most finishing aerospace technical activities of aircraft must be synchronized with corresponding ground stations $[1,2]$. This synchronous mode has a number of defects [3]. By contrast, the direct establishment of high-precision time frequency reference within the constellation can enhance the autonomous operational capability of aerospace application systems. Therefore, further studies on related theories and implementation technologies related to autonomous time synchronization are necessary to improve the precision of autonomous time synchronization of constellations.

Current domestic and international studies on autonomous time synchronization focus on the following aspects: autonomous navigation algorithms for satellite navigation systems, space-based measurement and control, space-based information transport architecture and routing algorithms, distributed satellite systems, new atomic frequency standards, and improved precision of existing time synchronization methods [4-9]. Current studies seldom focus on autonomous time synchronization algorithms and data processing models used in satellite constellations. Based on a simulation of the characteristics of satellite visibility time of a layered satellite constellation, this study focuses on an autonomous time synchronization algorithm and an improvement method that corresponds to its constellation structure. This study uses 
the two-way time synchronization algorithm to conduct simulated autonomous time synchronization between different layers of satellites.

\section{Satellite Constellation Autonomous Time Synchronization}

Satellite constellation autonomous time indicates that given a lack of long-term support from a ground system, the satellite constellation constantly updates the satellite clock parameters introduced by the ground station by using two-way distance measurements between satellites, data exchanges, and an on-board filtering processor to establish and maintain the constellation time [3]. The autonomous navigation ability of a constellation has been studied extensively, specifically under the precondition of malfunction or destruction of the ground measurement and control station. In such a case, the satellite equipment in the constellation can synchronize via intersatellite distance measurements combined with established satellite orbit information. Such measurements ensure precise orbit determination for autonomous operation, time reference maintenance, long-term independent operation of the constellation, reconstruction and repair of the measurement and control station, and enhancement of the antijamming and antidamage ability of the system. Autonomous time synchronization is a key technology for autonomous navigation constellations $[4,10]$. Only with autonomous time synchronization can autonomous satellite constellation navigation meet the required level of precision. Thus, autonomous time synchronization and time keeping technology is a major research topic in the field of aerospace application systems.

\section{Visual Simulation of a Layered Satellite Constellation}

A layered satellite constellation is composed of three types of orbiting satellites, each forming their own layer: geostationary earth orbit (GEO), inclined geosynchronous orbit (IGSO), and medium earth orbit (MEO). Intersatellite physical visibility must be achieved to ensure autonomous time synchronization in a layered satellite constellation. Electromagnetic power requirements must then be met to ensure normal communication during physical visibility. Therefore, considering the visual time modeling and simulation of the layered satellite, constellation is essential in studying autonomous time synchronization for layered satellite constellation.

3.1. Mutual-Visual Model of Satellites. Generally speaking, after two satellites rotating around the earth determine their positions in the air at a random time, these satellites can see each other only when both of them are higher than the tangential level on the surface of the earth. The most extreme situation is when two satellites are located at the externally tangential level simultaneously, as shown in Figure 1. Therefore, we can obtain a visual function to depict whether two

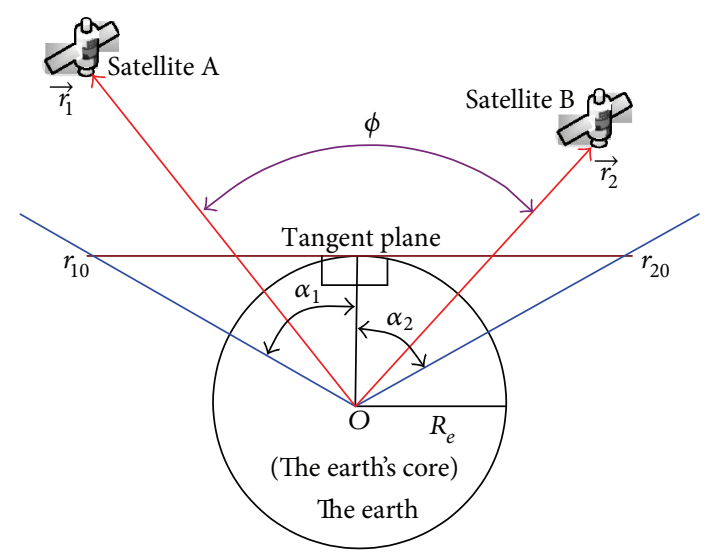

FIGURE 1: Visual calculation model between satellites.

TABLE 1: Comparison of mutual visual conditions for GEO, IGSO, and MEO satellites.

\begin{tabular}{lc}
\hline Link types & $\begin{array}{c}\text { Percentage of satellite } \\
\text { mutual-visual time accounting } \\
\text { for simulation cycle }\end{array}$ \\
\hline GEO-GEO & $100 \%$ \\
GEO-IGSO & $100 \%$ \\
GEO-MEO & $97.47 \%$ \\
IGSO-MEO & $98.55 \%$ \\
\hline
\end{tabular}

satellites are within sight of each other. This visibility function is as follows [11]:

$$
\psi=\alpha_{1}+\alpha_{2}-\phi
$$

where $\alpha_{1}$ and $\alpha_{2}$ are the limiting angles and $\phi$ represents an included angle between two position vectors of two satellites that connect to the core of the earth at different positions. The values of $\alpha_{1}, \alpha_{2}$, and $\phi$ are indicated in [11]. When $\psi>0$, the two satellites can see each other. Otherwise, they have no visual contact.

3.2. Mutual-Visual Simulation Results between Satellite Constellation Layers. Based on the mutual-visual model, the Satellite Tool Kit (STK) software is used to simulate typical visual conditions between satellites at different layers of a layered satellite constellation. The simulated statistical results are presented in Table 1.

Table 1 shows that GEO and IGSO satellites can see each other continuously for 24 hours each day. The visibility time of GEO and MEO satellites accounts for $97.47 \%$ of the entire MEO satellite regression cycle and that of IGSO and MEO satellites accounts for $98.55 \%$. Therefore, when the ground station cannot be used, we can conduct autonomous time synchronization by establishing satellite links in high rail among GEO, IGSO, and MEO satellites. The average synchronous time of MEO satellites can account for $98 \%$ of the simulation cycle to improve the autonomous time synchronization precision of layered constellations [12]. 


\section{Autonomous Time Synchronization Algorithm for a Layered Satellite Constellation}

According to the simulation results, when the ground station cannot be used, the constellation time is established uniformly, mainly through intersatellite mutual autonomous time synchronization. The accurate satellite position cannot be known beforehand, and precise measurement of the propagation path delay of the time signal between satellites is difficult to obtain. Therefore, the autonomous time synchronization algorithm of the constellation should significantly reduce the path propagation delay of the time synchronization signal, and the main measurement should be completed autonomously by the satellites.

4.1. Two-Way Time Synchronization Algorithm. Because the two-way time synchronization algorithm of the satellites does not require the accurate position of the two satellites in advance, the algorithm can offset the influence of the propagation path and other additional delays, which results in precise time synchronization. When the satellites can see each other in the constellation, the two-way time synchronization algorithm can be used directly for intersatellite autonomous time synchronization in the layered satellite constellation. The intersatellite two-way time synchronization principle is shown in Figure 2 [13]. Satellites A and B are installed with radio transmitters and receivers. Both satellites transmit and receive each others time synchronization signals simultaneously. The equation can be expressed as follows:

$$
\begin{gathered}
T_{1}=\Delta t+t_{2}+\tau_{\mathrm{BA}}+r_{1}+\delta_{1}, \\
T_{2}=-\Delta t+t_{1}+\tau_{\mathrm{AB}}+r_{2}+\delta_{2} .
\end{gathered}
$$

In (2), $\Delta t$ represents the clock correction of Satellites $\mathrm{A}$ and $\mathrm{B}, T_{1}$ is the time difference between Satellite $\mathrm{A}$ transmitting its timing signal and receiving the timing signal transmitted by Satellite B, $t_{2}$ indicates the transmitting equipment delay of Satellite B, $\tau_{\mathrm{BA}}$ indicates the propagation time delay from Satellite B to Satellite A, $r_{1}$ stands for the receiving equipment delay of Satellite $\mathrm{A}, \delta_{1}$ indicates other delays, $T_{2}$ represents the time difference between Satellite B transmitting its timing signal and receiving the timing signal transmitted by Satellite $\mathrm{A}, t_{1}$ is the transmitting equipment delay of Satellite $\mathrm{A}, \tau_{\mathrm{AB}}$ indicates the propagation time delay of from Satellite A to Satellite B, $r_{2}$ is the receiving equipment delay of Satellite B, and $\delta_{2}$ indicates other delays.

After solving (2), we can obtain the clock offset $\Delta t$ of Satellites A and B:

$$
\begin{aligned}
\Delta t= & \frac{T_{1}-T_{2}}{2}+\frac{t_{1}-t_{2}}{2}+\frac{r_{2}-r_{1}}{2} \\
& +\frac{\tau_{\mathrm{AB}}-\tau_{\mathrm{BA}}}{2}+\frac{\delta_{2}-\delta_{1}}{2} .
\end{aligned}
$$

In (3), $T_{1}$ and $T_{2}$ can be determined by measuring Satellites A and B. $t_{1}, r_{1}, t_{2}$, and $r_{2}$ can each be calibrated in advance according to the satellite transmission signal

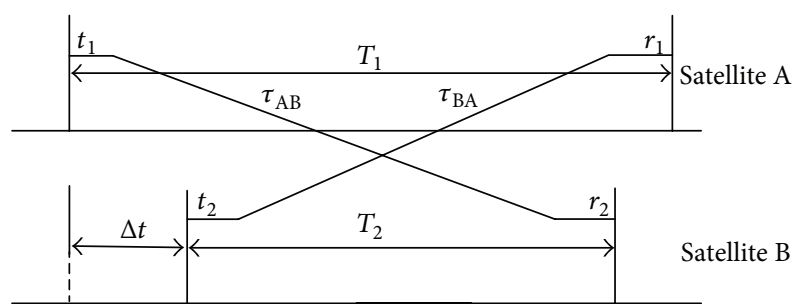

FIGURE 2: Schematic of two-way time synchronization of satellites.

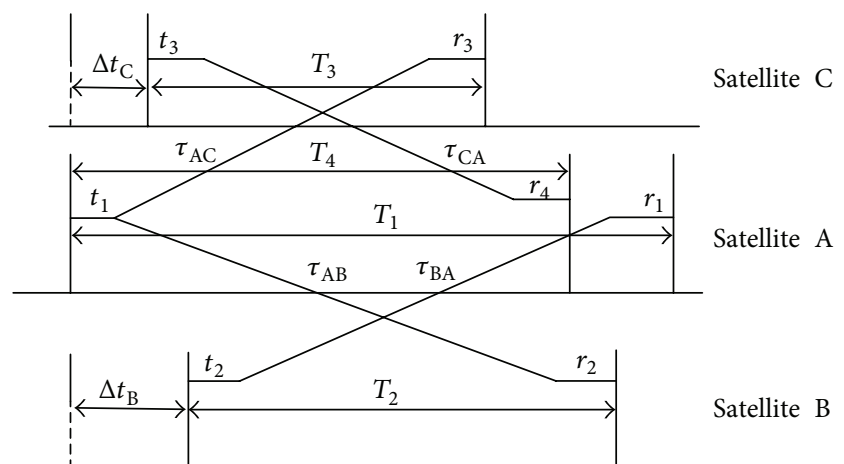

FIGURE 3: Schematic of common view two-way time synchronization.

frequency. When the frequencies of the time synchronization signals transmitted by Satellites A and B are close, the link is symmetrical and the propagation delay is approximately equal: $\tau_{\mathrm{AB}}=\tau_{\mathrm{BA}}$. Meanwhile, ignoring the effects of other delays, we can determine the satellite clock offset.

4.2. Common View Two-Way Time Synchronization Algorithm. If the satellites in a constellation cannot see each other, we cannot directly use the two-way time synchronization algorithm to achieve intersatellite autonomous time synchronization in the layered satellite constellation. Thus, we have to use a public visual satellite to complete the indirect twoway time synchronization, that is, common view two-way time synchronization, as shown in Figure 3. In the figure, Satellite A is a mutual visual satellite assumed to be either GEO or IGSO type. Satellites B and C cannot see each other. When Satellite A transmits the time synchronization signal to Satellites B and C simultaneously and receives the time synchronization signals of the other satellites, according to the principle of two-way time synchronization, the following equations are obtained:

$$
\begin{aligned}
\Delta t_{\mathrm{B}}= & \frac{T_{1}-T_{2}}{2}+\frac{t_{1}-t_{2}}{2}+\frac{r_{2}-r_{1}}{2} \\
& +\frac{\tau_{\mathrm{AB}}-\tau_{\mathrm{BA}}}{2}+\frac{\delta_{2}-\delta_{1}}{2} \\
\Delta t_{\mathrm{C}}= & \frac{T_{4}-T_{3}}{2}+\frac{t_{1}-t_{3}}{2}+\frac{r_{3}-r_{4}}{2} \\
& +\frac{\tau_{\mathrm{AC}}-\tau_{\mathrm{CA}}}{2}+\frac{\delta_{3}-\delta_{4}}{2} .
\end{aligned}
$$



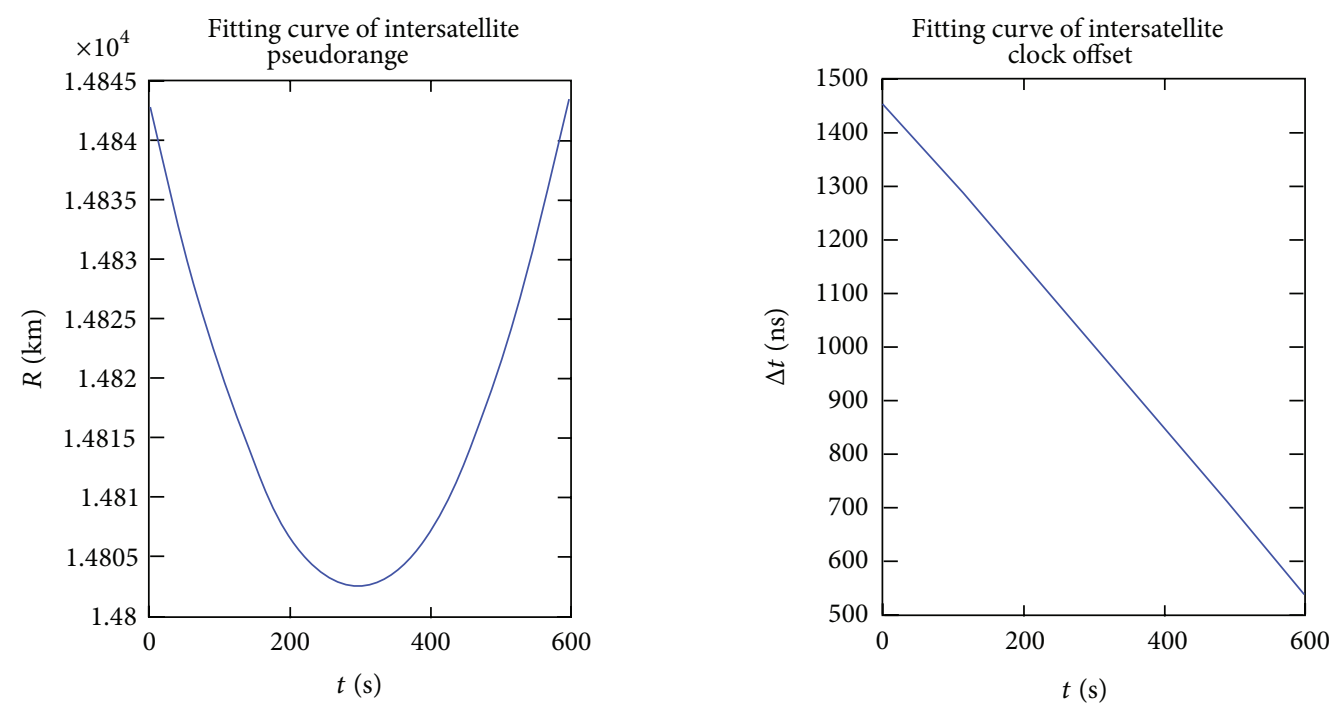

(a) Fitting results of a 10-minute time synchronization from 23:32:50.000 on June 1, 2008 to 23:42:50.000 on June 1, 2008
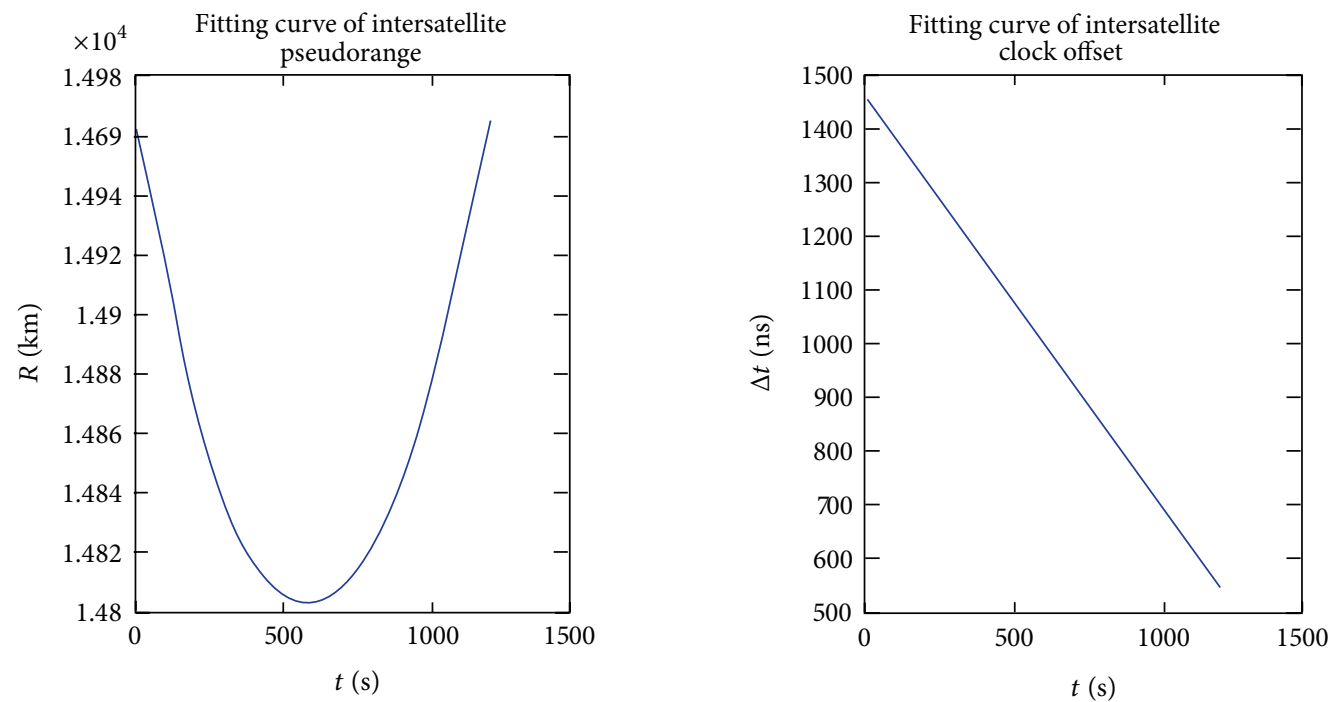

(b) Fitting results of a 20-minute time synchronization from 23:27:50.000 on June 1, 2008 to 23:47:50.000 on June 1, 2008
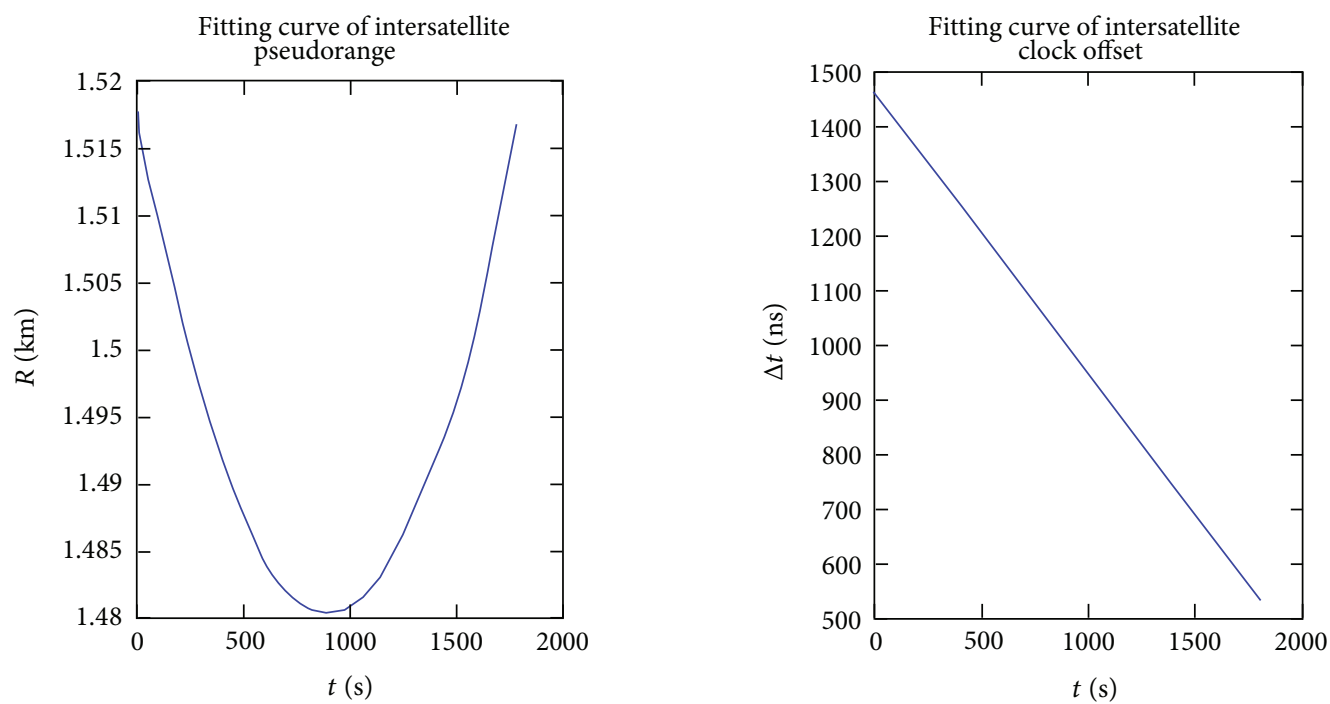

(c) Fitting results of a 30-minute time synchronization from 23:22:50.000 on June 1, 2008 to 23:52:50.000 on June 1, 2008

FIgURE 4: Continued. 

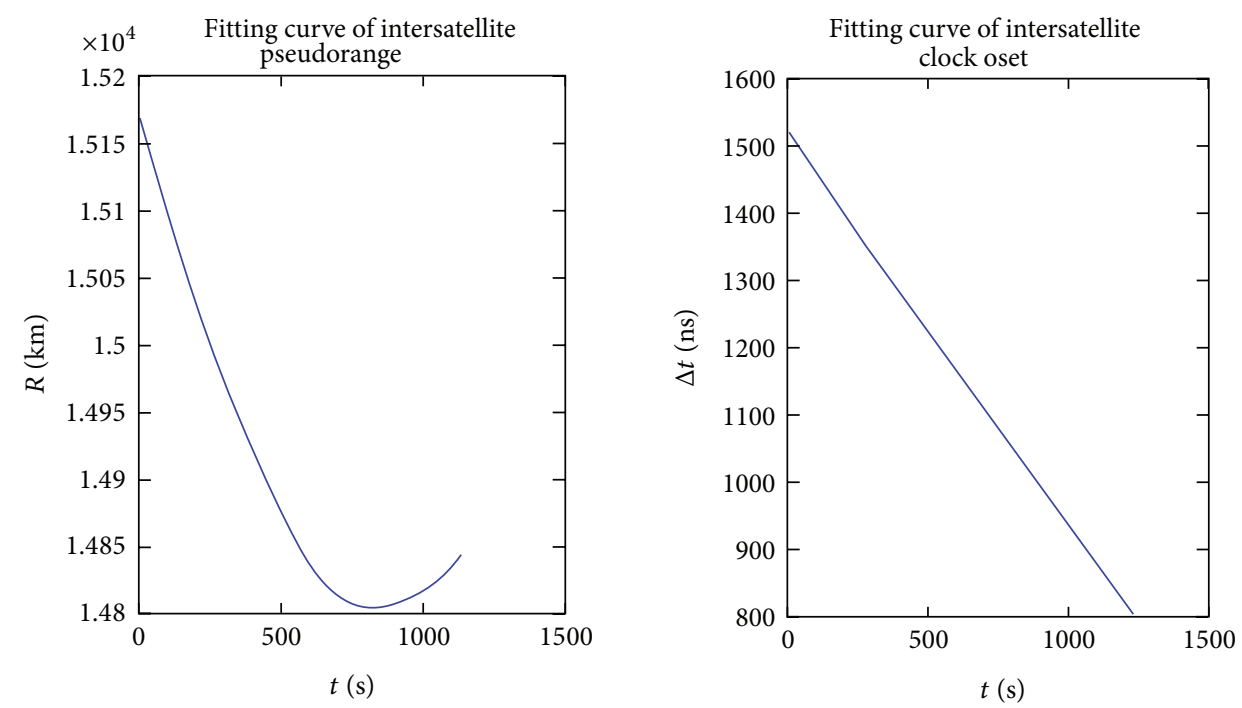

(d) Fitting results of a 20-minute time synchronization from 23:22:50.000 on June 1, 2008 to 23:42:50.000 on June 1, 2008 (asymmetrical)

FIGURE 4: Fitting curves of pseudorange and clock offset between GEO and MEO satellites.

In (4), $\Delta t_{\mathrm{B}}$ and $\Delta t_{\mathrm{C}}$ indicate the clock offset between Satellites A and B and between Satellites A and C, respectively. The other parameters have the same meanings as in two-way time synchronization.

After solving (4), we can obtain the clock offset $\Delta t_{\mathrm{BC}}$ of Satellites B and C as follows:

$$
\begin{aligned}
\Delta t_{\mathrm{BC}}= & \frac{T_{1}+T_{3}-T_{2}-T_{4}}{2}+\frac{t_{3}-t_{2}}{2}+\frac{r_{2}+r_{4}-r_{1}-r_{3}}{2} \\
& +\frac{\left(\tau_{\mathrm{AB}}-\tau_{\mathrm{BA}}\right)-\left(\tau_{\mathrm{AC}}-\tau_{\mathrm{CA}}\right)}{2}+\frac{\delta_{3}+\delta_{1}-\delta_{4}-\delta_{2}}{2} .
\end{aligned}
$$

In (5), the transmission time delay $t_{1}$ of mutual visual Satellite $\mathrm{A}$ is eliminated. With the receiving channel delay $r_{4}$ and $r_{1}$ of mutual visual Satellite $A$ are equal, and other delays $\delta_{1}$ and $\delta_{4}$ are the same, and the impact on time synchronization of those delay is eliminated. The transmission delays of Satellites B and C ( $t_{2}$ and $\left.t_{3}\right)$ receive delays $r_{2}$ and $r_{3}$ and can be calibrated in advance according to the satellite transmitting signal frequency. Disregarding other delays and the effects of $\delta_{2}$ and $\delta_{3}$, we can simplify (5) as follows:

$$
\Delta t_{\mathrm{BC}}=\frac{T_{1}+T_{3}-T_{2}-T_{4}}{2}+\frac{\left(\tau_{\mathrm{AB}}-\tau_{\mathrm{BA}}\right)-\left(\tau_{\mathrm{AC}}-\tau_{\mathrm{CA}}\right)}{2} .
$$

Considering $\tau_{\mathrm{AB}}=\tau_{\mathrm{BA}}$ and $\tau_{\mathrm{AC}}=\tau_{\mathrm{CA}}$ in (6), we have to determine only the time difference between each transmission timing signal of Satellites A and B and Satellites $\mathrm{A}$ and $\mathrm{C}$ measured by the satellite itself as well as the receiving timing signal transmitted by another satellite; thus, the clock offset of two satellites that cannot see each other is acquired accurately.

\section{Two-Way Time Synchronization Algorithm Improvement and Simulation}

5.1. Principle of Improved Two-Way Time Synchronization Algorithm. In the two-way time synchronization algorithm and common view time synchronization algorithm, determining the clock offset of mutually visual and invisible satellites requires taking advantage of the fact that the mutual transmission two-way time synchronization signals have approximately equal propagation delays. However, given the high speed of satellites in the constellation, the paths of the two-way time synchronization signals of the constellation generally do not have equal propagation delays, and the precision of the clock offset obtained by applying the algorithm is reduced. Thus, we have improved this algorithm so that it can be used in unequal delay conditions.

According to the movement characteristics of satellites in the constellation, the time synchronization can be conducted when the intersatellite distance has regular changes. We can use an intersatellite pseudorange fitting polynomial and an intersatellite clock offset polynomial to express the process of two-way time synchronization. The intersatellite pseudo range and clock offset polynomial after fitting are as follows [14]:

$$
\begin{aligned}
R & =f_{1}(t), \\
\Delta t & =f_{2}(t),
\end{aligned}
$$

where $R$ indicates the pseudo range polynomial and $\Delta t$ indicates the clock offset polynomial. If we suppose that $d f_{1}(t) / d t=0$, then we can determine the corresponding time $t_{3}$ when the intersatellite pseudo range is at its minimum value $R_{\min }$, that is, when the calculated intersatellite clock offset and the actual satellite clock offset are at the proximate moment. Substituting $t_{3}$ into (8), we can find 
TABLE 2: Comparison of least squares fitting results of different time lengths of the GEO and MEO satellites in the same period (on June 1, 2008 , the actual clock offset is $1 \mu \mathrm{s})$.

\begin{tabular}{|c|c|c|c|c|}
\hline Period & $23: 32: 50.00$ to $23: 42: 50.00$ & $23: 27: 50.00$ to $23: 47: 50.00$ & $23: 22: 50.00$ to $23: 52: 50.00$ & $\begin{array}{c}\text { 23:22:50.00 to } 23: 42: 50.00 \\
\text { (asymmetrical) }\end{array}$ \\
\hline Pseudorange & $R=0.0004620897 t^{2}$ & $R=0.0004601381 t^{2}$ & $R=0.0004569549 t^{2}$ & $R=0.0004594126 t^{2}$ \\
\hline fitting & $-0.2767866515 t$ & $-0.5525447643 t$ & $-0.8239841380 t$ & $-0.8273193074 t$ \\
\hline polynomial & +14843.9503933924 & +14968.4674054507 & +15174.4148791739 & +15175.1192383119 \\
\hline $\begin{array}{l}\text { Pseudorange } \\
\text { minimum } \\
\text { value } \\
\text { moment cor- } \\
\text { responding to } \\
\rho_{\min }(\mathrm{s})\end{array}$ & 299.4944839741193 & 600.411855223807 & 901.603341546627 & 900.4098268430367 \\
\hline $\begin{array}{l}\text { Clock offset } \\
\text { fitting } \\
\text { polynomial }\end{array}$ & $\begin{aligned} \Delta t= & -1.53961927325 t \\
& +1462.39429390793\end{aligned}$ & $\begin{aligned} \Delta t= & -0.77368332728 t \\
& +1464.46555576661\end{aligned}$ & $\begin{aligned} \Delta t= & -0.51949244881 t \\
& +1467.72268747967\end{aligned}$ & $\begin{aligned} & \Delta t=-0.58308473555 t \\
&+1505.84764677203 \\
&\end{aligned}$ \\
\hline $\begin{array}{l}\text { Minimum } \\
\text { clock offset } \\
\Delta t_{\min } \text { (ns) }\end{array}$ & 1001.286814148183 & 999.9369138812618 & 999.3465597276504 & 980.8324209975162 \\
\hline
\end{tabular}

the corresponding intersatellite clock offset with minimum error $\Delta t_{\min }=f_{2}\left(t_{3}\right)$ when the intersatellite pseudo range is at the minimum.

5.2. Improved Two-Way Time Synchronization Algorithm Simulation. To validate the accuracy of the improved twoway time synchronization algorithm, we apply it to the autonomous time synchronization between a GEO satellite (Satellite A) and an MEO satellite (Satellite B). If we assume that the actual clock offset of Satellites A and B is $1 \mu \mathrm{s}$ prior to synchronization, four sections of dynamic twoway time synchronization data are generated using STK. The equipment delay of the receiver and transmitter on the satellite as well as other delays are neglected. We can obtain the least squares fitting results of the pseudo range and clock offset of GEO and MEO satellites for different time lengths within the same period, as shown in Figure 4 and Table 2.

Figure 4 and Table 2 show that when the synchronization period is basically symmetrical to the moment when the minimum intersatellite range appears, the time synchronization and polynomial fitting are more precise, the fitting error is small, and the error is within the $1 \mathrm{~ns}$ to $2 \mathrm{~ns}$. However, when the synchronization period is not symmetrical to the moment when the minimum intersatellite range appears, the time synchronization and polynomial fitting are less precise, the fitting error is large, and the error reaches $20 \mathrm{~ns}$.

The pseudo range of the different time lengths and the clock offset simulation results of the GEO and MEO satellites in the same period show that the clock offset acquired using the improved two-way time synchronization algorithm is significantly close to the actual clock offset, and the improved two-way time synchronization model and algorithm are accurate.

Considering these simulation results, we use the improved two-way time synchronization algorithm for autonomous time synchronization of different layers within the constellation of satellites. The duration of the two-way synchronization should be basically symmetrical to the distance between the minimum satellite moment to reduce polynomial fitting error and improve time synchronization accuracy.

\section{Conclusions}

Considering the need for autonomous time synchronization of layered satellite constellations, we adapted a two-way time synchronization algorithm to layered satellite constellations and investigated a common view two-way time synchronization algorithm. The major effects of satellite motion on algorithm error were analyzed. The main measures of the effect of satellite motion on the algorithm were also investigated. Finally, an improved two-way time synchronization algorithm was applied to the autonomous time synchronization of the GEO-MEO satellite layer within a satellite constellation. Simulation results demonstrate that when the synchronization period is basically symmetrical to the moment when the minimum intersatellite range appears, and when least squares fitting pseudo ranges polynomial and clock offset polynomial are used relative to the conditions of the other error factors, the autonomous time synchronization accuracy of the algorithm can be controlled at less than $5 \mathrm{~ns}$. This result may be useful in conducting autonomous high-precision time synchronization in a layered satellite constellation.

\section{Conflict of Interests}

The authors declare that there is no conflict of interests.

\section{Acknowledgments}

This study was supported by the National Natural Science Foundation of China (nos. 11073022 and 10673011), the Hunan Provincial Natural Science Foundation (no. 11JJ3072), 
the Scientific Research Fund of the Hunan Provincial Education Department (nos. 13A115 and 11B015), the Science and Technology Planning Project of Changsha (no. K100801211), and the Open Fund for Key Laboratory of Precision Navigation and Timing Technology, National Time Service Center, Chinese Academy of Sciences (no. 2009PNTT07).

\section{References}

[1] H. L. Wu, S. R. Guo, and S. W. Fan, "The realization of twoway time synchronization between the satellite and the ground station in passive satellite navigation system," GNSS World China, vol. 6, pp. 36-40, 2003.

[2] S. S. Tan, "Two-way pseudo range time synchronization for navigation satellite clock," Engineering Science, vol. 8, no. 5, pp. 70-74, 2006

[3] H. T. Wu, X. H. Li, Y. Hua, X. C. Lu, and X. H. Yang, Time Foundation of Satellite Navigation System, chapter 6, Science Press, Beijing, China, 2011.

[4] P. Shuai, G. J. Qu, and Z. G. Chen, "Studies on autonomous navigation techniques for navigation constellations," Engineering Science, vol. 8, pp. 22-30, 2006.

[5] X. S. Sun, D. Yang, X. Yang, and K. Zhai, “The tracking laws of user satellite tracking TDRS," Journal of Astronautics, vol. 25, no. 1, pp. 41-45, 2004.

[6] J. J. Bai, X. C. Lu, Z. X. Lu, and W. Peng, "A distributed hierarchical routing protocol for Non-GEO satellite networks," in Proceedings of the International Conference on Parallel Processing Workshops, vol. 2, pp. 148-154, 2004.

[7] L. Shi, J. J. He, and S. W. Lu, "Model and simulation of space communication infrastructure," Journal of System Simulation, vol. 18, no. 6, pp. 1643-1652, 2006.

[8] S. J. Zhang and G. R. Duan, "Cooperative control for distributed satellite formation keeping," Journal of Astronautics, vol. 32, no. 10, pp. 2140-2145, 2011.

[9] Z. C. Zhai, "Recent progress in atomic time and frequency," World Sci-Tech R and D, vol. 28, no. 3, pp. 63-69, 2006.

[10] P. Shuai and G. J. Qu, “Time synchronization techniques of the autonomous navigation of navigation constellation," Journal of Astronautics, vol. 26, no. 6, pp. 768-772, 2005.

[11] G. X. Zhang and H. Zhang, Satellite Mobile Communication System, chapter 2, Beijing, China, 2001.

[12] Q. F. Xu, "Satellite constellation of local navigation system," Engineering of Surveying and Mapping, vol. 10, no. 1, pp. 1-6, 2001.

[13] F. J. Huang, X. C. Lu, H. T. Wu, and Y. J. Bian, "Study on autonomous time synchronization method based on intersatellite," Computer Science, vol. 35, no. 9, pp. 123-125, 2008.

[14] F. Huang, X. Lu, H. Wu, and Y. Bian, "An algorithm of dynamic two-way time transfer based on intersatellite range variation," Geomatics and Information Science of Wuhan University, vol. 35, no. 1, pp. 13-16, 2010. 


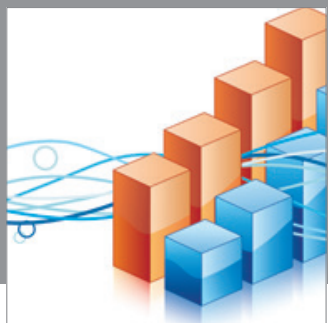

Advances in

Operations Research

mansans

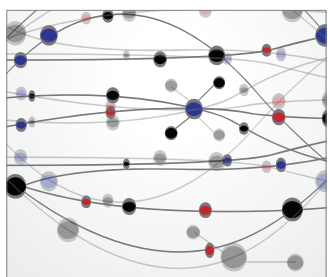

The Scientific World Journal
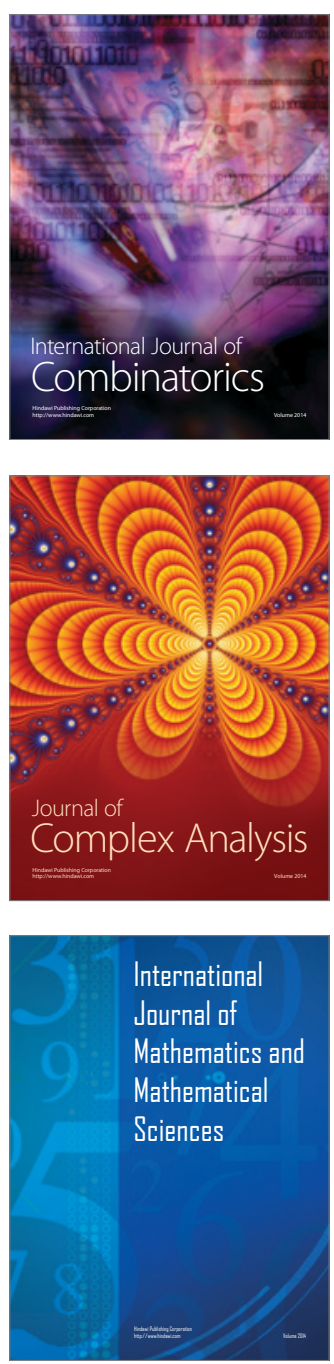
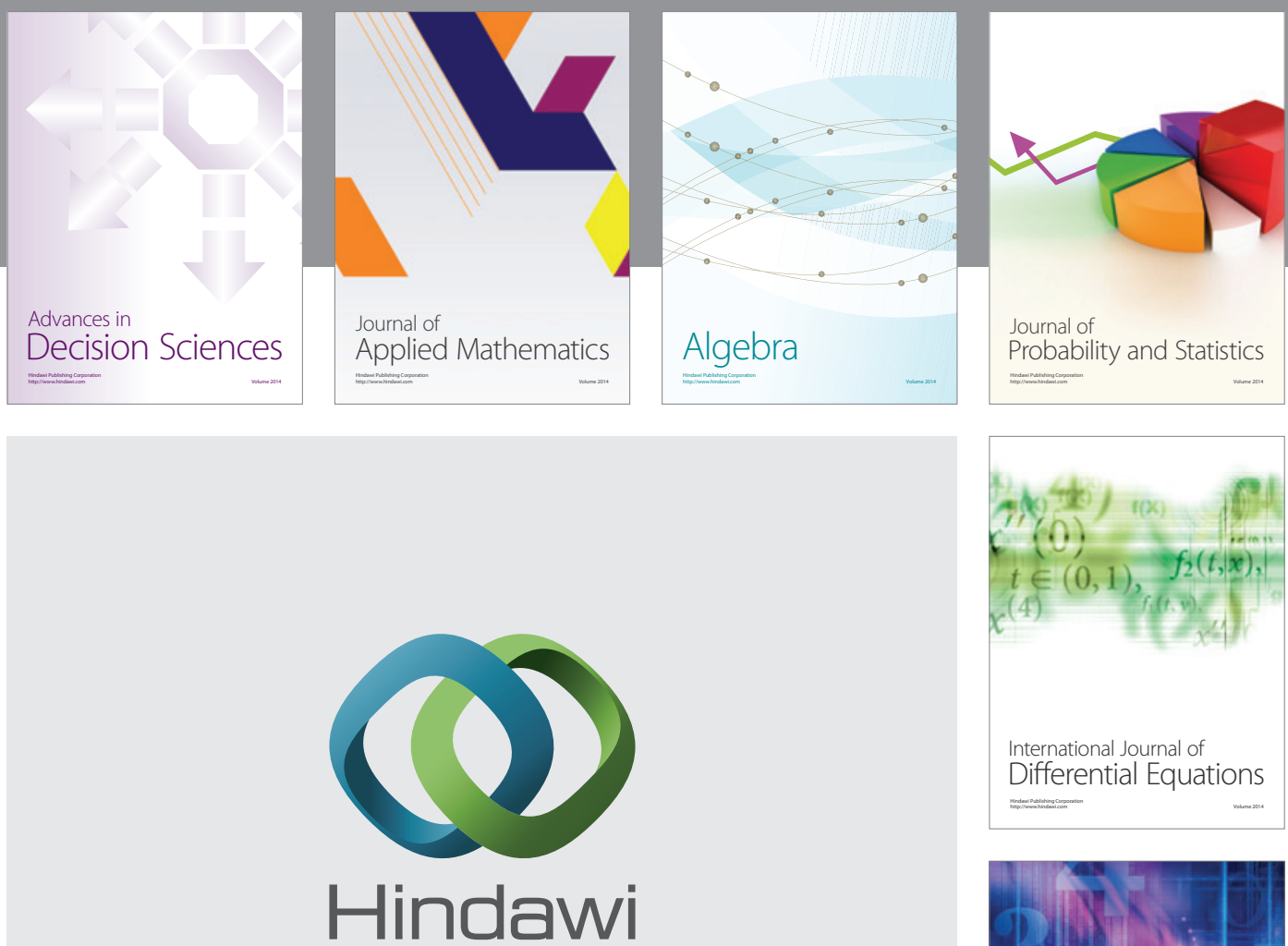

Submit your manuscripts at http://www.hindawi.com
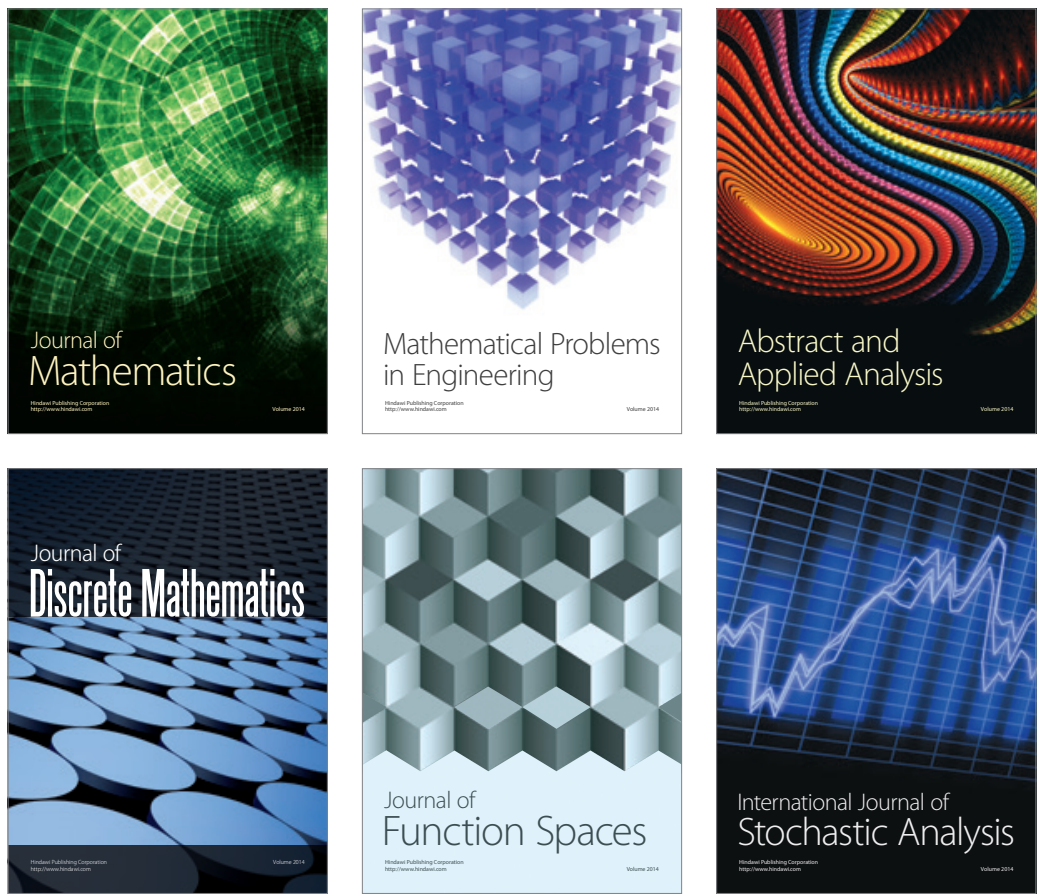

Journal of

Function Spaces

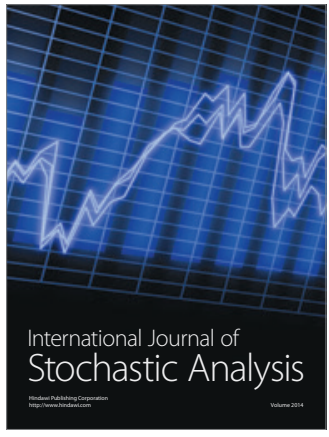

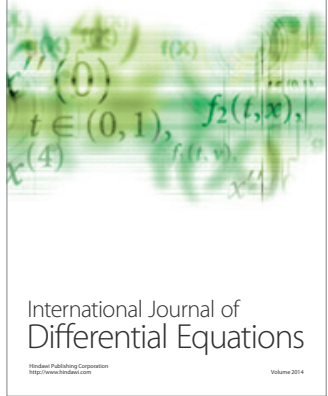
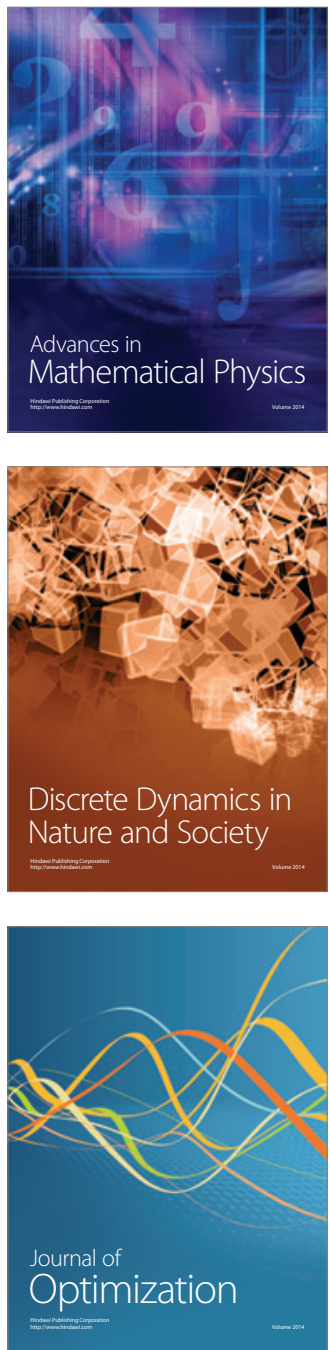